\title{
DYSLEXIA TREATED BY HEMISPHERE STIMULATION TECHNIC
}

Results of treatment of severe dyslexia in 80 children, ages 6 to 15 years, using hemisphere stimulation technics, are reported from the outpatient Department for Dyslexia, Child Psychiatric Center, Paedological Institute, Amsterdam, The Netherlands. Treatment was tailored for each child, depending on the type of dyslexia, according to the Bakker (1990) P/L classification as follows: $P$ type - right hemisphere readers, using perceptual-spatial features of text (slow, fragmented, but relatively accurate); L type - left hemisphere preference, using lingual (semantic and syntactic) aspects of text (inaccurate, hasty, reading technic). About $40 \%$ are unclassified, but an inappropriate or nonoptimal hemisphere preference is considered the basis for the dyslexia in the majority of subjects. For dyslexics with an inaccurate, hasty reading strategy, right-hemisphere $(\mathrm{RH})$ stimulation technics were employed. For the non fluent, slow readers, left-hemisphere (LH) stimulation was used. The very slow readers who had not mastered grapheme-phoneme conversion received a short period of home mediation therapy to facilitate automatic mastery of letter-sound correspondence. Tactile methods, using plastic lettered text, were employed for stimulation of the right hemisphere, and visual half-field presentation of words, flashed on the right side of a television screen, for stimulation of the left hemisphere. Auditory stimulation was presented as words in one ear and non-verbal (music) in the other, in order to reduce activation of the ipsilateral hemisphere. Twelve levels of text difficulty were selected individually for training. The average duration of treatment was 63 weeks (52 hours). Whereas treatment with flash cards and automatic lettersound conversions had a slight benefit, hemisphere stimulation technics produced significant improvements in single-word and text reading. (Kappers, EJ. Outpatient treatment of dyslexia through stimulation of the cerebral hemispheres. I Learning Disabilities Jan/Feb 1997;30:100-125). (Dr E Jan Kappers died Oct 23, 1995. Manuscript revisions suggested by reviewers and provided by Dr Victor HP van Daal and Dr Dirk J Bakker).

COMMENT. This hemisphere classification and neuropsychological treatment of dyslexia was formulated by Bakker DJ (Oxford University Press, New York, 1990; I Learning Disabilities 1992;25:102-109; and earlier related publications). Bakker reported most favorable results with right-hemisphere stimulation, using tactile methods, whereas Kappers found equal benefit from right- and left-hemisphere stimulation technics, presented according to the dyslexic sub-type. The majority of dyslexics required left hemisphere stimulation after a period of initial right hemisphere stimulation. Very poor readers benefit from practice of grapheme-phoneme (letter-sound) conversions. Training of letter-sound correspondences is more successful than teaching of phonemic awareness in severe dyslexics. Boys and girls were affected equally by treatment, despite reported differences in the functional organization of the brain for language development.

\section{Language-associated cortical regions are proportionally}

larger in the female brain, according to a stereological neuropathological examination of adult brains of 10 males and 11 females at the University of Sydney, and the Prince of Wales Medical Research Institute, Randwick, Australia. (Harasty KJ, Double KL, Halliday GM, et al. Arch Neurol Feb 1997;54:171-176). Females have larger Wernicke (superior temporal gyrus) and Broca (inferior frontal gyrus) language-associated regions compared with males, correlating with superior language skills attributed to females. 